\title{
Aircraft Platform Temperature Prediction Algorithm For Unsteady State Process
}

\author{
Chang Haijuan \\ Quality Engineering Center \\ China Aero-Polytechnology Establishment \\ Beijing, China \\ changhaijuan@163.com
}

\author{
Pang Liping \\ School of Aviation Science and Engineering \\ Beijing University of Aeronautics and Astronautics \\ Beijing, China \\ pangliping@buaa.edu.cn
}

\begin{abstract}
Aircraft platform environment has an important impaction on the aboard equipment performance. In all environment factors, the temperature is an key one because it will affect the working lifespan and operational performance of aircraft. Therefore, it is necessary to study the temperature prediction algorithm for an aircraft platform. Based on the error caused by the reasonableness of the assumptions and verified in the further, an aircraft platform temperature prediction algorithm for unsteady state process was studied in this paper. Firstly, based on unsteady state process, combined with energy conservation law, a lumped parameter model was established. Then the coefficients of model was estimated by using Kalman filtering, and the average temperature in the front equipment cabin was predicted for given flight profiles. From simulation results, it can be seen that the unsteady state process temperature prediction method is able to tack the variation of average temperature of front equipment cabin. This method provides a new idea for aircraft platform temperature prediction.
\end{abstract}

Keywords-temperature prediction; filtering estimation; unsteady state process; environmental worthiness

\section{INTRODUCTION}

Platform environment, which impact equipment's performance and sometimes induce malfunctions, severely affect the lifespan and operational performance of aircraft and its airborne equipment, worse still, may affect safety of aircraft. As a statistic by America: 52 percent of the malfunctions and invalidation were caused by environment, still more, 74 percent of these malfunctions were caused by induced vibration, shock, temperature and humidity environment. Therefore, platform environment prediction has become hotspot concerned by developed countries. Mission environment requirement integrated technique (MERIT), which based on interactive expert system, combined with some algorithm and rule, can predict the environmental stress of full life cycle profile ${ }^{[1]}$.

From the 1990's, domestic environmental engineering expert began research the technology of environment prediction. Most research of environment prediction algorithm focus on the vibration environment, and only few research involved temperature prediction algorithm. Even the research on temperature prediction algorithm just focused on the method to build the temperature model by statistical approach based on the great quantity of measured data. At present, the common algorithm including the multi-linear regression method and parameter optimization method based on physical model ${ }^{2 \sim 3]}$. But the former didn't consider the physical reality, as well, the latter which based on the steady state thermal balance equation, didn't consider the process that temperature varies as time, therefore the prediction results of both algorithms are inaccuracy ${ }^{[4]}$. In addition, the temperature value measured by sensors may be influenced by noise ${ }^{[5]}$, but the traditional algorithms do not fully consider the influence of noise, therefore an algorithm of temperature prediction based on unsteady process was proposed in this paper.

\section{MODEL OF TEMPERATURE PREDICTION}

For subsonic speed aircraft, to get the temperature of unsealed equipment cabin, we can take front equipment cabin as a macrocosm, then, radiating equipment becomes the main heat producer, meantime, air-conditioning and ram-air becomes the main cold producer. Make the following assumptions:

a. For subsonic speed aircraft, the Mach number normally is less than 1 , so the temperature variation in equipment cabin caused by aerodynamic heating can be ignored;

b. There is no glazing in front equipment cabin, and this cabin is under pilot cabin, therefore, solar radiation can be ignored;

c. There is thermal-protective coating between equipment cabin and other cabins, therefore, the convection, radiation and heat conduction between equipment cabin and other cabins can be ignored;

d. Cooling air flow of air-conditioning system is invariable.

Based on above assumptions, from conservation of energy, we can get the following equation ${ }^{[6 \sim 8]}$.

$$
\begin{gathered}
\rho(t) \cdot V \cdot C_{p} \cdot \frac{\partial T}{\partial t}=P(t)+\Delta Q \cdot C_{p} \cdot\left(T_{i n}(t)-T_{t}\right) \\
+\Delta m \cdot C_{p} \cdot\left(T_{s}(t)-T_{t}\right)
\end{gathered}
$$

where $\rho$ is air density in equipment cabin at time $t ; V$ represents cubage of equipment cabin; $C_{p}$ is constantpressure mass specific heat of air in equipment cabin; $T$ 
represents average temperature in equipment cabin; $\Delta Q$ is mass flow of cooling airflow from air-conditioning system; $\Delta m$ is mass flow of ram-air; $T_{\text {in }}$ is inlet air temperature of air-conditioning system at time $t ; T_{\mathrm{s}}$ is recovery temperature at time $t ; P$ represents radiating power of electronic equipment in equipment cabin at time $t$.

In Eq. (1), the term, $\rho(t) \cdot V \cdot C_{p} \cdot \frac{\partial T}{\partial t}$, represents heat increment rate in equipment cabin at time $t$; $\Delta Q \cdot C_{p} \cdot\left(T_{i n}(t)-T_{t}\right)$ represents heat entrapped by airconditioning system at time $t ; \Delta m \cdot C_{p} \cdot\left(T_{S}(t)-T_{t}\right)$ represents heat entrapped through heat transfer between equipment cabin and environment at time $t$.

Then, the implicit discrete equation of Eq.(1) is written as:

$$
\frac{T_{t+1}-T_{t}}{\Delta t}=\frac{P(t)}{\rho(t) V C_{p}}+\frac{\Delta Q}{\rho(t) V}\left(T_{\text {in }}(t)-T_{t}\right)+\frac{\Delta m}{\rho(t) V}\left(T_{s}-T_{t}\right)
$$

Then we get the following Eq.

$$
\begin{aligned}
T_{t+1}= & \left(1-\frac{(\Delta Q+\Delta m) \cdot \Delta t}{\rho(t) V}\right) T_{t}+\frac{P(t) \cdot \Delta t}{\rho(t) V C_{p}} \\
+ & \frac{\Delta Q \cdot \Delta t}{\rho(t) V} T_{i n}(t)+\frac{\Delta m \cdot \Delta t}{\rho(t) V} T_{S}
\end{aligned}
$$

It can be written as

$$
T_{t+1}=A(t) \cdot T_{t}+\frac{\Delta m \cdot \Delta t}{\rho(t) V} T_{S}+\frac{B(t)}{\rho(t)}
$$

where

$$
\begin{gathered}
A(t)=1-\frac{(\Delta Q+\Delta m) \cdot \Delta t}{\rho(t) V} \\
B(t)=\frac{P(t) \cdot \Delta t}{V C_{p}}+\frac{\Delta Q \cdot \Delta t}{V} T_{i n}(t)
\end{gathered}
$$

Considering the rounding error during discrete and the model error caused by kinds of assumption during modeling, it's necessary to add a stochastic disturbance to system, then, Eq. (4) can be written as

$$
T_{t+1}=A(t) \cdot T_{t}+\frac{\Delta m \cdot \Delta t}{\rho(t) V} T_{s}+\frac{B(t)}{\rho(t)}+U_{1} \cdot \omega_{1}
$$

where, $\omega_{1}$ is Gaussian color noise, $E\left[\omega_{1} \cdot \omega_{1}^{\mathrm{T}}\right]=Q ; Q$ represents model noise, the more accurate the model is, the lower the number of $Q$ is; $U_{1}$ represents the coefficient of noise.

Given the mass flow of cooling airflow from airconditioning system, mass flow of ram-air, and cubage of equipment cabin, the function of air density in unhermetical cabin can be calculated through measured air data, then, combined Eq.(5), coefficient $A(t)$ can be calculated. Coefficient $B(t)$ is unknown because the inlet temperature of air-conditioning system and radiating power of electronic equipment via time is unknown. Therefore, to calculate $B(t)$, Kalman Filter algorithm is introduced in references [9] and [10].

The state equation is as follows:

$$
\begin{gathered}
T_{t+1}=A(t) \cdot T_{t}+\frac{\Delta m \cdot \Delta t}{\rho(t) V} T_{s}+\frac{B(t)}{\rho(t)}+U_{1} \cdot \omega_{1} \\
B(t+1)=B(t)+U_{2} \cdot \omega_{2}
\end{gathered}
$$

It can be written as

$$
X_{t+1}=\Phi(t) X_{t}+b+U \cdot \omega
$$

where $\omega=\left(\omega_{1}, \omega_{2}\right)^{T}, X_{t}=\left(T_{t} B_{t}\right)^{T}, \quad \Phi(t)=\left(\begin{array}{cc}A(t) & 1 / \rho(t) \\ 0 & 1\end{array}\right)$, $b=\left(\begin{array}{ll}\frac{\Delta m \cdot \Delta t}{\rho(t) V} T_{s} & 0\end{array}\right)^{T}$.

The observation equation is

$$
Z(t)=\left[\begin{array}{ll}
1 & 0
\end{array}\right] \cdot X_{t}
$$

Taking $H=\left[\begin{array}{ll}1 & 0\end{array}\right]$, then Kalman one step optimum preestimate equation is

$$
\hat{Y}_{t+1 \mid t}=\Phi(t) \cdot X_{t}+b_{t}
$$

One step optimum pre-estimate error is

$$
P_{t+1 \mid t}=\Phi(t) P_{t \mid t} \Phi(t)^{T}+\Gamma(t) Q_{t} \Gamma(t)^{T}
$$

The gain is

$$
K_{t+1}=P_{t+1 \mid t} H^{T}\left[H P_{t+1 \mid t} H^{T}+R\right]^{-1}
$$

The optimum filter estimate equation is

$$
X_{t+1}=\dot{Y}_{t+1 \mid t}+K_{t+1}\left(Z(t+1)-H \cdot Y_{t+1 \mid t}\right)
$$

Filter estimate error is

$$
\begin{gathered}
P_{t+1 \mid t+1}=\left(I-K_{t+1} H\right) P_{t+1 \mid t}\left(I-K_{t+1} H\right)^{T} \\
+K_{t+1} R K_{t+1}^{T}
\end{gathered}
$$




\section{SIMULATION OF MODEL}

Take a certain aircraft as example, the mass flow of ram-air is constant when aircraft fly at a certain height. Then from Eq. (5), coefficient $A(t)$ is constant. Once more, making an assumption that the inlet temperature of airconditioning system keep invariable when aircraft fly at a certain height and at a certain velocity, as well, radiating power of electronic equipment in equipment cabin keep invariable, then, from Eq. (6), coefficient $B(t)$ is constant, thus, we get

$$
A(t)=\frac{T_{t+k+1}-T_{t+1}}{T_{t+k}-T_{t}}
$$

According to temperature and air data measured during flying at a Shanghai airport on September to December, 2002, where the first flight of 17th September, there is nearly 500 s flying at a constant velocity at height $3900 \mathrm{~m}$, intercepting the measured data at this time slice, combining with Eq.(15), coefficient $A(t)$ when flying at height $3900 \mathrm{~m}$ can be calculated. Meanwhile, density $\rho(t)$ can be calculated by interpolation method, then, we can get $A(t)$ of the whole flight. Still more, recovery temperature can be calculated based on interpolation of air data and weather data, then, we get $B(t)$.

From Kalman filtering algorithm, coefficient $B(t)$ can be estimated and average temperature of equipment cabin can be predicted, the result is shown in Fig .1 and Fig .2.
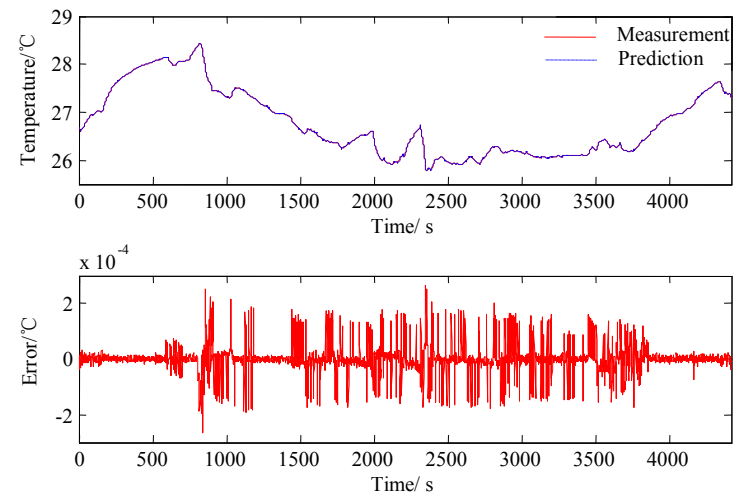

Figure 1. Temperature filter esimation of front equipment cabin.

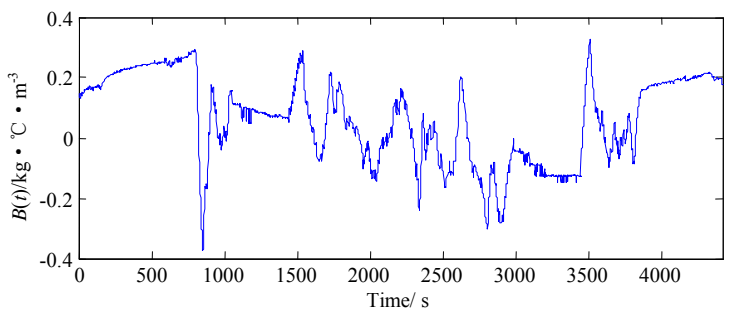

Figure 2. Esitmation result of coefficient $B(t)$.

The temperature in front equipment cabin on halt mode is affected by ground surface temperature, airflow speed, solar radiation intensity, as well as type of barrier, coefficient of heat transfer and so on. Thus, based on measured data on halt mode, input estimated $B(t)$ into Eq.(4), given flight profile as Fig .3, we can get the temperature curve of front equipment cabin as shown in Fig.4.
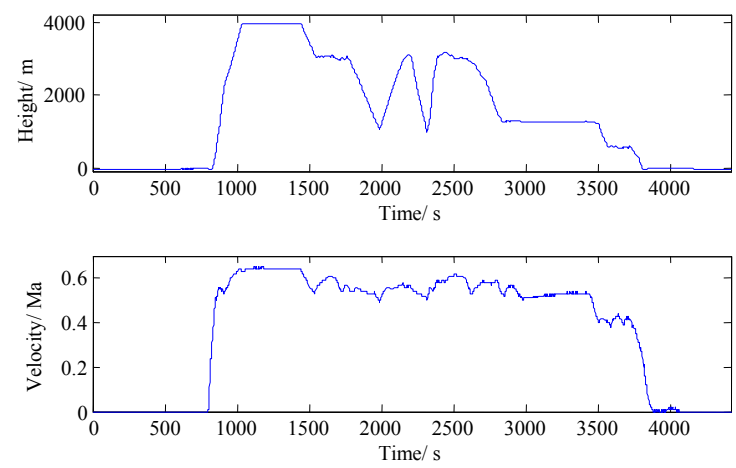

Figure 3. Flight profile.
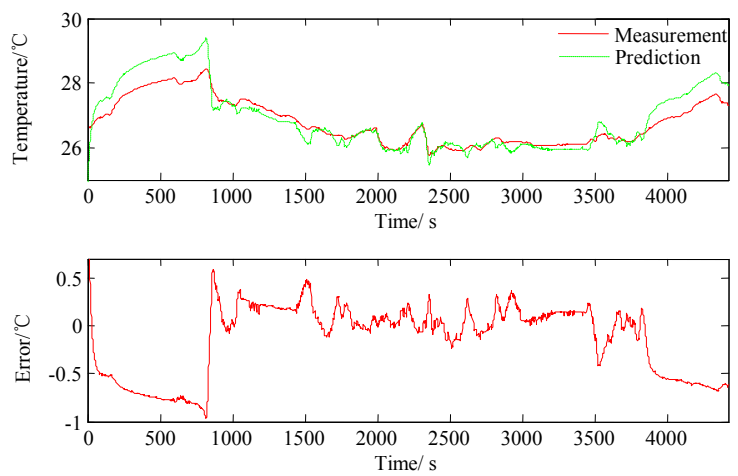

Figure 4. Temperature prediction results of front equipment cabin.

As shown in Fig .4, the algorithm advanced in this paper can accurately predict temperature curve of front equipment cabin, though predict error of taking off segment and slide after landing segment is relatively higher.

Then, we validate the accuracy of this algorithm with measured data of the second flight in the same day. The second flight was low altitude fly. The highest height of which was $1000 \mathrm{~m}$, and the flight profile was shown in Fig .5. Based on the estimated $B(t)$, average temperature of front equipment cabin at this flight profile can be predicted, as is shown in Fig .6.

It's obvious that Fig .6 is similar to Fig .4. Firstly, the predict error of front equipment cabin is almost the same between two flight profile; secondly, it can be seen from these two figure, the predict error of the algorithm is relatively bigger during taking off segment and landing segment. 

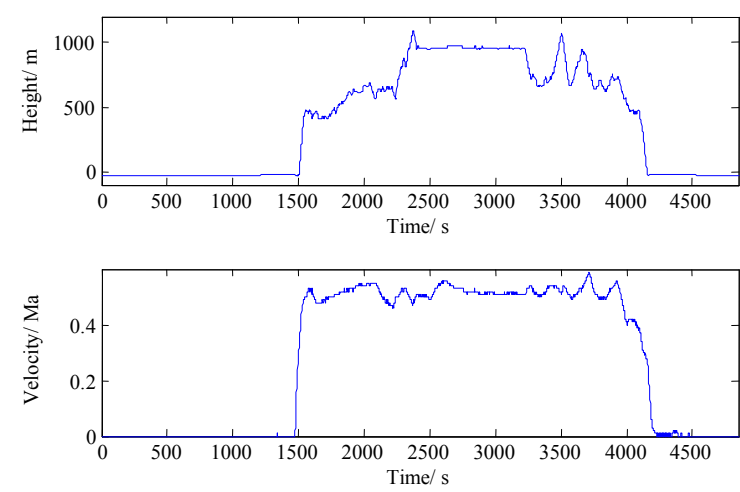

Figure 5. Flight profile.
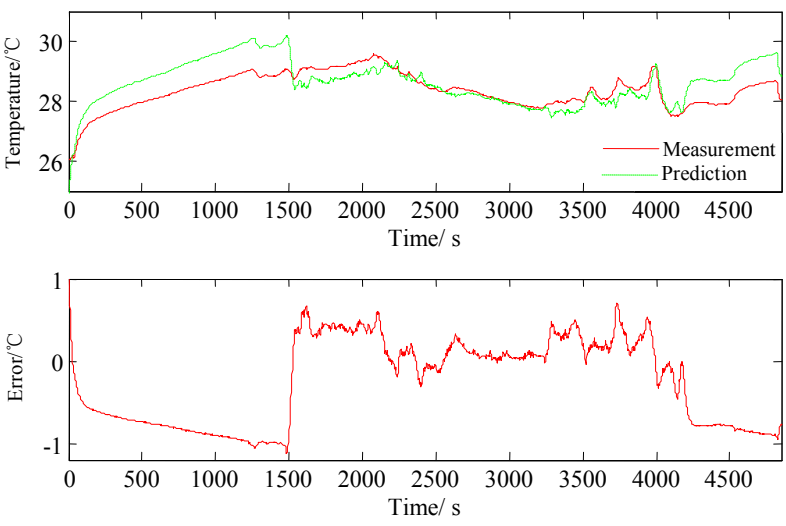

Figure 6. Temperature prediction results of front equipment cabin.

\section{RESULT ANALYSIS}

This article proposed a temperature prediction method for unsteady state process using lumped parameter model combined with energy conservation law. Using Kalman filtering model coefficients were estimated, and average temperature of front equipment cabin was predicted. From simulation results, it can be seen that this method is more consistent to physical practice and has less prediction errors compared with traditional linear regression method or physical model based parameter optimization method. This method may not be very accurate for equipment cabin temperature prediction during aircraft ground taxing phase (including takeoff and landing). The possible reasons might be two points: Firstly, part of model assumptions does not apply to the ground starting, taxing, takeoff, landing, landing roll etc. (e.g. ram air cooling flow rate constant does not apply to ground pneumatic and roll case); Secondly, environmental control system boot time may not be estimated properly, and this article assumes that aircraft environmental control system is always running during the whole process. Follow-up study will focus on the mathematical model of flight profile segment (takeoff and landing phase of flight stage). The temperature prediction algorithm is expected to further improve in order to improve the accuracy.

The algorithm in this article is built on the basis of variable assumption, model assumption, and the error caused by the reasonableness of the assumptions remains to be further verified. This article only provides a new idea of the temperature prediction. Welcome further comments and recommendations of relevant experts, to discuss and build a mature temperature prediction algorithm.

\section{ACKNOWLEDGMENT}

The research is sponsored by the Aviation Science Foundation of China (20130251005).

\section{REFERENCES}

[1] Xia Xin-lin, Ai Qing, Ren De-peng, "Numerical analysis on the transient thermal status of aircraft". Acta Aeronautica Et Astronautica SinicA, vol. 28(3), pp. 513-518, 2007.

[2] P. Sarangi and et al., "Short Term Load Forecasting using Artificial Neural Network: A Comparison with Genetic Algorithm Implementation", J. of ARPN Engineering and Applied Sciences, vol. 9, 2009.

[3] S. Singh, P. Bhambri, J. Gill, Time series based temperature prediction using back propagation with genetic algorithm technique, IJCSI International Journal of Computer Science Issues, vol 8(5), pp. 1694-0814, 2011.

[4] Paul Heaton, Jeffrey Czuchna, "Prediction of dynamic environments for airborne external stores during aircraft straight and level flight," Journal of the Institute of Environmental Sciences, vol. 39(4), pp. 28-32, 1996.

[5] J. Gill, B. Singh and S. Singh, "Training Back Propagation Neural Networks with Genetic Algorithm for Weather Forecasting", 8th IEEE International Symposium on Intelligent Systems Subotica Serbia, 2010, vol. 8, pp. 465-469.

[6] Tao Wen-quan, Numerical heat transfer, 2nd ed., Xi'an: Xian Jiaotong University press, 2006, pp.80-95.

[7] R. Vilaithong, S. Tenbohlen, T. Stirl, Neural network for transfrmer top-oil temperature prediction, XVth International symposium on high voltage engineering, August, 27-31, 2007.

[8] J.K. Pylvanainen, K. Nousiainen, P. Verho, "Studies to utilize loading guides and ann for oil-immersed distribution transforme condition monitoring", IEEE Trans, Power Delivery, vol. 22, pp. 201-207, 2007.

[9] Chen Peng, Wang Yan-dong, Foundermental theory of modern contral, 1st ed. Beijing: Beihang Unversity press, 2004, pp. 78-95.

[10] Wang Qiu-ping, Chen Juan, Wang Xian-li, "New adaptive nonlinear kalman filters algorithm," Opto-Electronic Engineering, vol. $35(7), 2008$. 